\title{
Foxo3a aggravates inflammation and induces apoptosis in IL-1-treated rabbit chondrocytes via positively regulating tenascin-c
}

\author{
Fei Wang1, Qiubin Wang', Ming Zhu ${ }^{2}$, Qi Sun ${ }^{1}$ \\ ${ }^{1}$ Department of Rehabilitation Medicine, The First People's Hospital of Huzhou City, Huzhou City, \\ Zhejiang Province, 313000, China \\ ${ }^{2}$ Department of Nephrology, The First People's Hospital of Huzhou City, Huzhou City, Zhejiang \\ Province, 313000, China
}

\begin{abstract}
Introduction. Osteoarthritis (OA) is the most common degenerative disease in middle-aged and elderly individuals that causes joint deformity and limb disability. Accumulating evidence has suggested that the pathogenesis of OA has been related to various mechanisms such as apoptosis, inflammation, oxidative stress and metabolic disorders. The aim of this study is to clarify the role of Foxo3a in the progress of OA in an in vitro model.

Materials and methods. The chondrocytes were derived from rabbit, and treated with IL- $1 \beta$, which was used as an in vitro OA model. The over-expression and down-regulation of Foxo3a were achieved by transfection with overexpression vector or shRNA, respectively. The mRNA level of iNOS in chondrocytes was quantified by qPCR. Tenascin-c (Tnc) production was measured by ELISA and apoptosis-associated proteins were analyzed by Western blotting. The MTT assay was used to assess the viability of chondrocytes.

Results. Foxo3a and iNOS expression were upregulated in IL- $1 \beta$-treated chondrocytes. Foxo3a silencing decreased iNOS expression, and inhibited apoptosis of IL- $1 \beta$-treated chondrocytes. The production of Tnc was significantly increased in IL- $1 \beta$-treated chondrocytes and was positively regulated by Foxo3. Importantly, extracellular addition of Tnc abrogated the protective effects of Foxo3a knockdown on IL- $1 \beta$ - treated chondrocytes. Conclusion. The present study indicated that down-regulation of Foxo3a protected IL- $1 \beta$-treated chondrocytes by decreasing iNOS expression and suppressing chondrocytes' apoptosis via modulating tenascin-c, which could be regarded as a potent therapeutic target for the treatment of OA. (Folia Histochemica et Cytobiologica 2020, Vol. 58, No. 1, 1-8)
\end{abstract}

Key words: chondrocytes; IL-1 $\beta$; osteoarthritis in vitro; Foxo3a; iNOS; tenascin-c; apoptosis; shRNA

\section{Introduction}

Osteoarthritis (OA) is known as a degenerative disease that gradually causes joint deformity and limb disability, which further causes a reduction in the quality of life and increases mortality [1]. OA is one of

Correspondence address: Qiubin Wang

Department of Rehabilitation Medicine,

The First People's Hospital of Huzhou City,

302, Unit B, Building 41, Jin shiji ming cheng,

Wuxing District, Huzhou City, Zhejiang Province, China tel.: 86+0572-257-5067

e-mail: QiubinWangawe@163.com the most common diseases in middle-aged and elderly individuals. In particular, the incidence of OA is more than $60 \%$ among people over 65 years of age [2]. Once the disease occurs, a balance of extracellular matrix (ECM) synthesis/degradation is broken and ultimately leads to the destruction of joint tissue [3]. Accumulating evidence suggests that the pathogenesis of OA is linked with various mechanisms. At the cellular level, apoptosis of articular chondrocytes and articular cartilage tissue cells is induced by several factors including cytokines, chemokines, Toll-like receptor ligands, and other inflammatory mediators such as nitric oxide [4]. At the molecular level, reactive oxygen species (ROS)-mediated oxidative stress signaling in 
chondrocytes, as well as mitochondrial dysfunction, metabolic disorders and other abnormal molecular signals are involved in the massive loss of cartilage, which are characteristics of OA [5]. Non-steroidal anti-inflammatory drugs (NSAIDs), the most common clinical treatment for OA, cause several serious side effects like peptic ulcer, nervous system dysfunction and bleeding after long-term use [6].

Tenascin-c (Tnc) is a member of the growing family of ECM proteins, and is expressed in normal adult tissues [7]. However, it is re-expressed during healing wounds, inflammatory responses and tumorigenesis $[8,9]$. For instance, a high level of Tnc was found in cartilage, synovial tissue and synovial fluid [10]. It was also reported that Tnc promotes the proliferation and migration of cells during both OA and rheumatoid arthritis [11]. Midwood et al., found that Tnc is involved in maintaining inflammation under arthritic joint disease [12].

Foxo3a, a Forkhead transcription factor of Forkhead box, class $\mathrm{O}$ (Foxo) subfamily, has been recently studied extensively as a critical protein that involved in the regulation of inflammation [13]. In arthritis, an increase in the expression as well as phosphorylation of Foxo3a in the blood of patients with rheumatoid arthritis was reported [14]. However, the exact role of Foxo3a in the pathogenesis of OA remains unclear. Therefore, understanding underlying mechanism of OA is helpful to explore effective molecular targets of this disease. Hence, the aim of this study was to clarify the role of Foxo3a in the progress of OA in an in vitro model of this disease.

\section{Materials and methods}

Animals and chondrocytes isolation. The one-year old New Zealand white rabbits were purchased from Shanghai SLAC Laboratory Animal Co., Ltd (Shanghai, China). All procedures were approved by the Medical Ethics Committee of the First Affiliated Hospital of Huzhou Normal University (Approval no. 2019008). The rabbits were housed at room temperature $\left(23-27^{\circ} \mathrm{C}\right)$ with a $12 \mathrm{~h}$ light: $12 \mathrm{~h}$ dark cycle, and the food and water were provided ad libitum. Prior to the animal experiment, rabbits were anesthetized with isoflurane, and then the articular cartilage was carefully harvested from knee joints by sterile dissection. Cartilage was cut into pieces as thin as possible (approximately $1 \mathrm{~mm}^{3}$ ) using SLICE!T® (Dr. Khan's Creation, Maharashtra, India), followed by digesting with enriched DMEM medium containing collagenase type II (Sigma-Aldrich Chemical Co, St. Louis, MO, USA) at the concentration of $2 \mathrm{mg} / \mathrm{mL}$ for $24 \mathrm{~h}$ at $37^{\circ} \mathrm{C}$.

Primary chondrocytes' culture and treatment. After collagenase digestion, isolated chondrocytes were washed with
Dulbecco's modified Eagle's medium (DMEM) (Gibco, Carlsbad, CA, USA) supplemented with $10 \%$ fetal bovine serum (FBS) (Gibco) and $1 \%$ penicillin/streptomycin $(0.1 \mathrm{mg} / \mathrm{mL}$ and $100 \mathrm{Units} / \mathrm{mL}(\mathrm{P} / \mathrm{S}, \mathrm{Gibco})$ and cultured in $5 \% \mathrm{CO}_{2}$ humidified incubator at $37^{\circ} \mathrm{C}$. The chondrocytes were cultured until the 3 rd passage and were used for the induction of OA in vitro model by adding to the isolated chondrocytes recombinant IL-1 $\beta$ (Sigma-Aldrich) at the concentration of $100 \mathrm{ng} / \mathrm{mL}$ for $48 \mathrm{~h}$ (based on a review by Johnson et al. [15]).

Plasmid construction. The lentiviral short hairpin RNA (shRNA) expression constructs to silence Foxo3a (Foxo3a-shRNA) were designed using the program published online by Invitrogen (Thermo Fisher Scientific, Waltham, MA, USA). The designed Foxo3a-siRNA-expressed vector was generated by Shanghai Genechem Co., Ltd (Shanghai, China). The shRNA strands were: sense 5'-AUCUAACUCAUCUGCAAGUUU-3'; anti-sense: 5'-ACUUGCAGAUGAGUUAGAUUU-3'. As the negative control for shFoxo3a, shNC was constructed. HA-Foxo3a WT was a gift from Michael Greenberg (Addgene plasmid \#1787; http://n2t.net/addgene:1787; RRID: Addgene_1787). The plasmid was amplified by EcoRI-XbaI into Flag tagged pcDNA3.1 retroviral backbone to develop pcDNA3.1-Foxo3a. pcDNA3. 1-NC was generated as a control.

Transfection. The chondrocytes were plated in 96-well plates at $10^{4}$ cells per well overnight, followed by transfection with either shRNA-Foxo3a or Foxo3a overexpression vector using Lipofectamine 2000 (Invitrogen, Thermo Fisher Scientific) according to the manufacturer's instructions. The recombinant Tnc $(1 \mu \mathrm{M})$ (Sigma-Aldrich) was added to the medium when chondrocytes were transfected.

RNA isolation, cDNA synthesis and quantitative PCR (qPCR). Total RNA from chondrocytes was extracted by TRIzol reagent (Invitrogen) following the instruction provided by the manufacturer. The RNA was reversely transcribed into complementary DNAs (cDNAs) using SuperScript IV First-Strand Synthesis System (Invitrogen) according to the manufacturer's instruction. The reverse transcription was carried out at $50^{\circ} \mathrm{C}$ for $1 \mathrm{~h}$, and then 1 unit of RNase H (Takara, Dalian, China) was treated at $37^{\circ} \mathrm{C}$ for $15 \mathrm{~min}$. The amplification conditions include a total of 30 cycles of pre-denaturation for $3 \mathrm{~min}$ at $94^{\circ} \mathrm{C}$, followed by denaturation for $30 \mathrm{sec}$ at $94^{\circ} \mathrm{C}$, annealing at $58^{\circ} \mathrm{C}$ for $30 \mathrm{sec}$ and extension for $60 \mathrm{sec}$ at $72^{\circ} \mathrm{C}$. The expression of inducible nitric oxide synthase (iNOS) was measured by SYBR-Green Master Mix (Applied Biosystems, Waltham, MA, USA) on ABIViiA7 Real-Time PCR System (Applied Biosystems). GAPDH was used as internal control for gene analysis.

The primer sequences were as follow: iNOS: 5'-CTGTGACGTCCAGCGCTACA-3', 5'-GCACGGCGATGTTGATCTCTCGCCT-3; GAPDH: 5'-GGAGAAAGCTGCTAA -3', 5'-ACGACCTGGTCCTCGGTGTA-3'. 


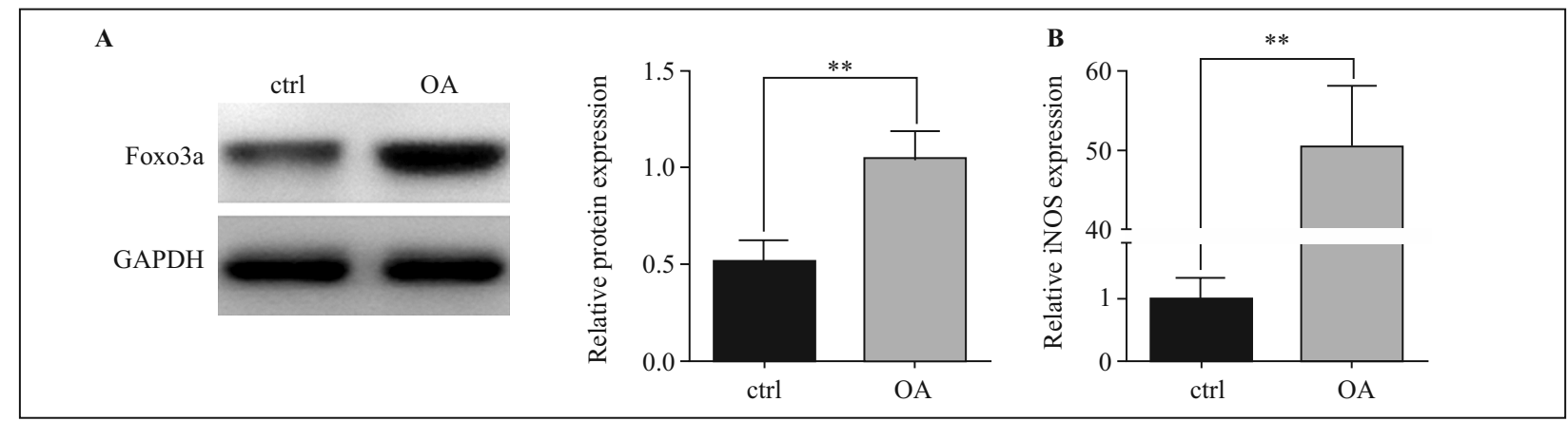

Figure 1. Foxo3a and iNOS were upregulated in IL-1 $\beta$-treated chondrocytes. The chondrocytes derived from rabbit knee joints were stimulated with IL-1 $\beta(100 \mathrm{ng} / \mathrm{mL})$ for $48 \mathrm{~h}$. The protein level of Foxo3a and mRNA level of iNOS were determined by western blotting (A) and qPCR (B), respectively. The data are presented as mean \pm SD $(n=3)$. Symbols: 'ctrl' - untreated, control chondrocytes, 'OA' - IL- $1 \beta$-treated chondrocytes. ${ }^{* *} \mathrm{p}<0.01$, OA vs. control.

Western blotting. The proteins from chondrocytes were lysed by radioimmunoprecipitation assay (RIPA) buffer. Protein assay was then used to quantify the concentration using a BCA Protein Assay Kit (Thermo Fisher Scientific). Fifty micrograms of protein were subjected to SDS-PAGE electrophoresis, and separated proteins were transferred to polyvinylidene difluoride (PVDF) membrane (Immobilon-P; EMD Millipore, Darmstadt, Germany). After that, the membrane was blocked with $5 \%$ of skill milk in TBS-T for $1 \mathrm{~h}$ and then incubated with 1000 time-diluted primary antibodies against Foxo3a (ARP38041-P050, Aviva Systems Biology, San Diego, CA, USA), Bax (OAED00050, Aviva Systems Biology), Bcl-2 (LSC465574-100, LifeSpan BioSciences, Inc, Seattle, WA, USA) and cleaved Caspase-3 (NB600-1235, Novus Biologicals, LCC Centennial, CO, USA) overnight at $4^{\circ} \mathrm{C}$. After washing three times in TBS-T, the membrane was incubated with secondary antibody for $1 \mathrm{~h}$ at room temperature. The GAPDH, as an internal control, was measured by anti-GAPDH(GTX100118, GENETEX, Inc) and its secondary antibody. Protein expression was detected using Luminata Forte Western HRP Substrate (Millipore) with a Bio-Rad ChemiDox XRS+ imaging system (Bio-Rad Laboratories, Hercules, CA, USA).

Cell viability. The viability of chondrocytes was detected using 3-(4,5)-dimethylthiahiazo(-z-yl)-3,5-di-phe-nyterazolium-bromide (MTT) (Gibco) assay based on mitochondrial reduction of MTT to formazan. Chondrocytes were pre-seeded in 96-well plate at a density of $10^{4}$ cells/well. Next day, the chondrocytes were transfected with or without shRNA or vectors in the presence of recombinant human IL-1 $\beta(100 \mathrm{ng} / \mathrm{mL})$. After incubation for $48 \mathrm{~h}$, the medium was replaced with $200 \mu \mathrm{L}$ of fresh medium. Then, $3 \mathrm{mg} / \mathrm{mL}$ of MTT in PBS was added into each well $(20 \mu \mathrm{L} /$ well $)$. After incubation for $1.5 \mathrm{~h}, 150 \mu \mathrm{L}$ of culture supernatant was removed from each well and the formazan crystal were lysed by $100 \mu \mathrm{L}$ of MTT stop solution $(0.4 \% \mathrm{HCl}, 10 \%$ Triton X-100 in isopropanol). After incubation for $12 \mathrm{~h}$, the absorbance was measured at $570 \mathrm{~nm}$ with $655 \mathrm{~nm}$ as reference wavelength on a microplate reader (Bio-Rad).

Measurement of Tnc level. Tnc production was measured by rabbit ELISA kit (MyBioSource, Inc, San Diego, CA, USA) according to the manufacturer's instruction. Briefly, diluted samples or standard recombinant tenascin were added into 96-well plates coated with anti-Tnc antibody for $2 \mathrm{~h}$ at room temperature. After washing, the detection antibody reacted at room temperature for $2 \mathrm{~h}$, followed by addition of an avidin-horseradish peroxidase conjugates to bind captured antigen. The reaction was measured by the absorbance at $490 \mathrm{~nm}$.

Statistical analysis. All data were expressed as means \pm standard deviation (SD). Data for each experiment were acquired from at least three independent experiments. Statistical analyses were performed by using Student's t-test via GraphPad Prism 7 (GraphPad Software, Inc., San Diego, CA, USA), with the level of significance set at $\mathrm{p}<0.05$.

\section{Results}

\section{Foxo3a and iNOS were upregulated in IL-1 $\beta$-treated chondrocytes}

As shown in Figure 1A, Foxo3a protein expression in IL- $1 \beta$-treated chondrocytes was significantly increased compared with control chondrocytes. Importantly, the mRNA expression of inducible nitric oxide synthase (iNOS), a biomarker of severe cellular stress, in IL-1 $\beta$-treated chondrocytes was also higher than that in control chondrocytes (Fig. 1B).

\section{Foxo3a knockdown inhibited IL-1 $\beta$-treated apoptosis of chondrocytes}

To further investigate the mechanism underlying the role of Foxo3a in the development of OA in our in 


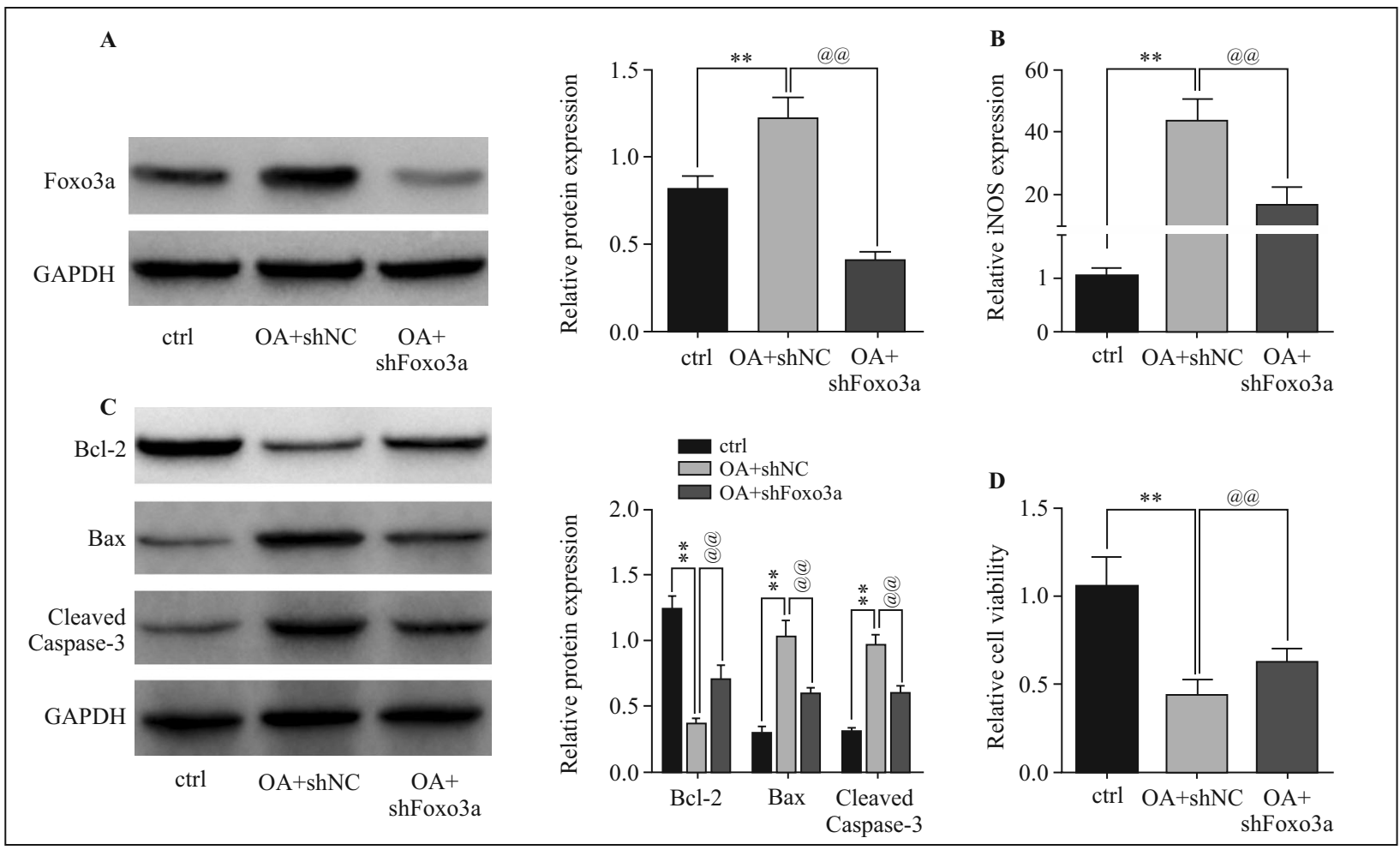

Figure 2. Foxo3a knockdown inhibited IL- $1 \beta$-treated apoptosis of chondrocytes. The chondrocytes derived from rabbit were stimulated with IL-1 $\beta(100 \mathrm{ng} / \mathrm{mL})$, followed by treatment with shNC or shFoxo3a for $48 \mathrm{~h}$. The protein level of Foxo3a and mRNA level of iNOS were determined by western blotting (A) and qPCR (B), respectively. The expression of apoptosis-associated proteins including bcl-2, bax and caspase-3 were analyzed by western blotting (C). The cell viability was measured by MTT assay (D). The data are presented as mean \pm SD $(n=3)$. Symbols as in the description of Figure $1 .{ }^{* *} p<0.01$, OA vs. control; ${ }^{@} \mathrm{p}<0.01, \mathrm{OA}+$ Sh-Foxo3a vs. OA + Sh-NC.

vitro model, we knocked-down Foxo3a expression in IL-1 $\beta$-treated chondrocytes by shRNA transfection. It was found that shRNA treatment strongly reduced Foxo3a protein expression in IL- $1 \beta$-treated chondrocytes (Fig. 2A). qPCR assay revealed that Foxo3a knockdown significantly decreased IL-1 $\beta$ -treated iNOS expression (Fig. 2B). The reduction of anti-apoptotic protein $\mathrm{Bcl}-2$ and enhanced levels of pro-apoptotic proteins, i.e. cleaved caspase- 3 and Bax, were observed in IL-1 $\beta$-treated chondrocytes (Fig. 2C). Interestingly, Foxo3a knockdown up-regulated Bcl-2 expression and decreased the proteins levels of Bax and cleaved caspase- 3 in IL- $1 \beta$-treated chondrocytes as compared with cells transfected with shRNA (Fig. 2C). MTT assay analysis showed that Foxo3a knockdown prompted the decreased viability caused by IL- $1 \beta$ in chondrocytes (Fig. 2D). Thus, these data suggested that Foxo3a knockdown promotes IL-1-induced apoptosis of chondrocytes.

\section{Foxo3a positively regulates Tnc expression}

The level of Tnc was upregulated in IL- $1 \beta$-treated chondrocytes as compared to the control (Fig. 3A). As shown in Figure 3B, the level of Tnc in IL- $1 \beta$-treated chondrocytes was dramatically decreased after shRNA-Foxo3a transfection (Fig. 3B). In contrast, the level of Tnc was significantly enhanced when Foxo3a was overexpressed in IL-1 $\beta$-treated chondrocytes (Fig. 3B).

\section{The protective effects of Foxo3a knockdown on IL-1-treated chondrocytes were reversed by extracellular Tnc}

To further evaluate whether Tnc was involved in the protective effects of Foxo3a knockdown in IL- $1 \beta$-treated chondrocytes, Tnc was added extracellularly to the cultures of IL- $1 \beta$-treated chondrocytes. It was found that the level of Tnc was appreciably increased in the presence of extracellular Tnc in IL- $1 \beta$-treated chondrocytes that were transfected with Foxo3a shRNA (Fig. 4A). The decreased expression levels of iNOS, Bax and cleaved caspase-3 caused by Foxo3a knockdown were rescued by the treatment with extracellular Tnc (Figs. 4B and 4C). In contrast, shFoxo3a-enhanced Bcl-2 expression was significantly declined in the presence of extracellular Tnc in IL-1 $\beta$-treated chondrocytes (Fig. 4C). It should 


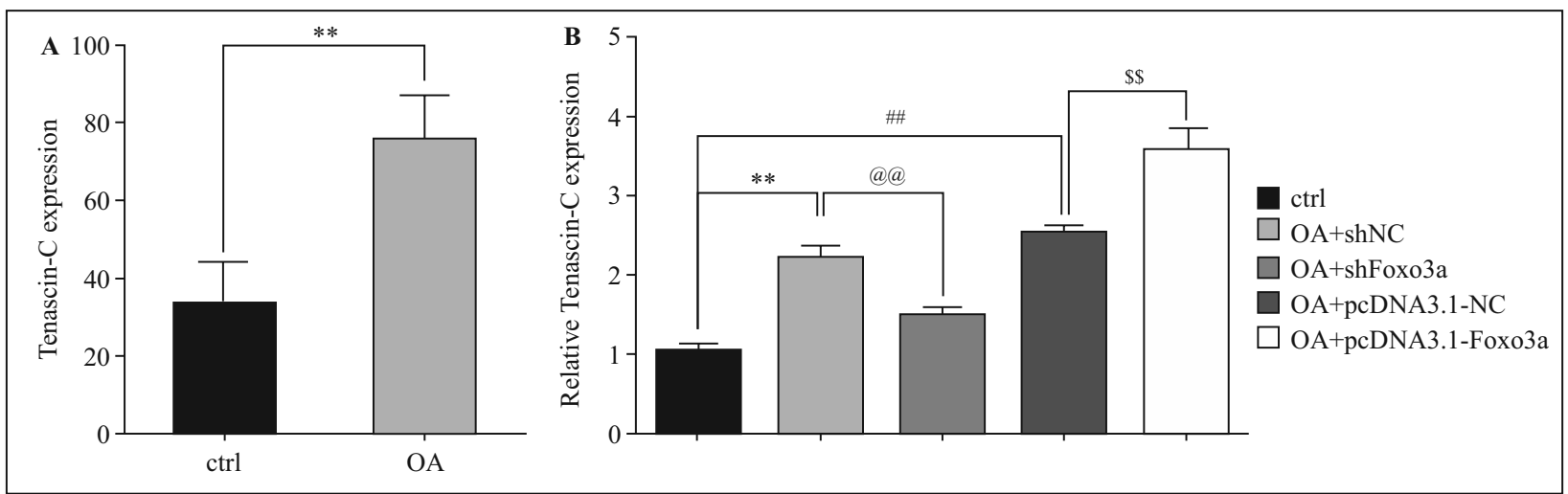

Figure 3. The expression of tenascin-c was positively regulated by Foxo3a. The chondrocytes were treated by IL-1 $\beta$ (100 ng/ $/ \mathrm{mL}$ ) for $48 \mathrm{~h}$. The level of tenascin-c in culture medium was quantified by ELISA (A). The IL-1 $\beta$-treated chondrocytes were transfected with shRNAs or vectors for $48 \mathrm{~h}$. The release of tenascin-c was measured by ELISA (B). The data were represented as mean $\pm \mathrm{SD}(\mathrm{n}=3)$. Symbols as in the description of Figure $1 .{ }^{* *} \mathrm{p}<0.01, \mathrm{OA}+\mathrm{shNC} v s$. control; ${ }^{\circledR} @ \mathrm{p}<0.01$, $\mathrm{OA}+$ shFoxo3a $v$ s. OA + shNC; ${ }^{\# \#} \mathrm{p}<0.01$, OA + pcDNA3.1-NC vs. control; ${ }^{\$} \mathrm{p}<0.01$, OA + pcDNA3.1-Foxo3a $v s$. Foxo3a OA + pcDNA3.1-NC.

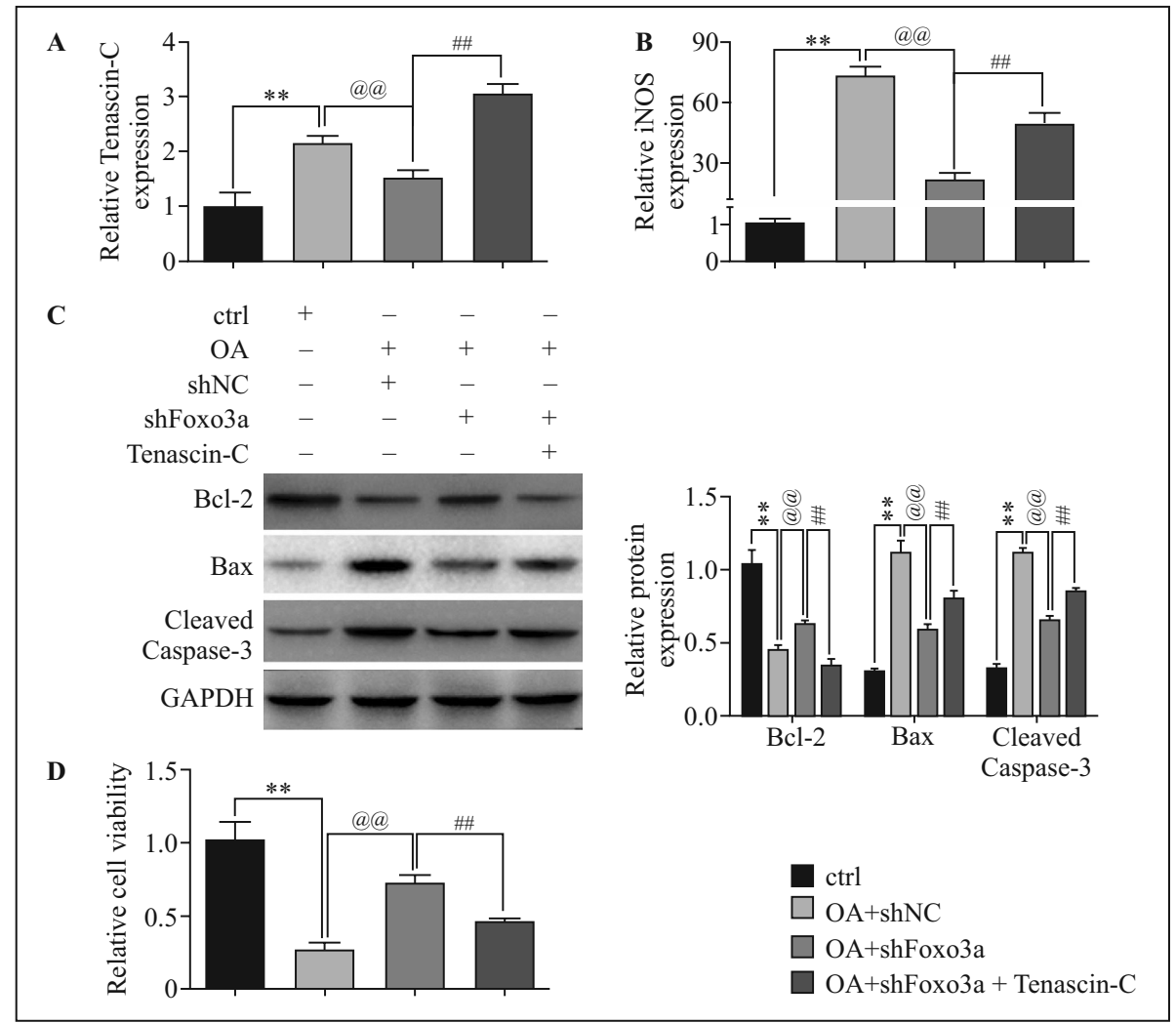

Figure 4. Extracellular tenascin-c abrogated the protective effect of down-regulated Foxo3a on IL- $1 \beta$-treated chondrocytes. The chondrocytes were stimulated with IL-1 $\beta(100 \mathrm{ng} / \mathrm{mL})$, followed by treatment of shFoxo3a in the absence or presence of tenascin-c $(1 \mu \mathrm{M})$. The level of tenascin-c in medium and mRNA level of iNOS were determined by ELISA (A) and qPCR (B), respectively. The expression of apoptosis-associated proteins was analyzed by western blotting (C), and the cell viability was measured by MTT assay (D). The data were represented as mean $\pm \mathrm{SD}(\mathrm{n}=3)$. Symbols as in the description of Figure 1. ${ }^{* *} \mathrm{p}<0.01, \mathrm{OA}+\operatorname{shNC} v s$. control; ${ }^{\circledR}{ }^{\circledR} \mathrm{p}<0.01, \mathrm{OA}+\operatorname{shFoxo3a} v s$. OA + shNC; ${ }^{\#} \mathrm{p}<0.01, \mathrm{OA}+\mathrm{shFoxo3a}$ + tenascin-c vs. OA + shFoxo3a. 
be noted that Tnc treatment effectively inhibited the increased cell viability caused by shFoxo3a in IL- $1 \beta$-treated chondrocytes (Fig. 4D). The evidence suggested that Foxo3a, as a positive regulator of Tnc, contributed to the apoptosis of IL- $1 \beta$-treated chondrocytes.

\section{Discussion}

Currently, the treatment strategies for OA mainly involve relieving the symptoms such as joints' pain, swelling, and muscle tension to improve the quality of life. NSAIDs, prescribed for a long-term use, are currently the primary pharmacological OA treatment. However, some dangerous side effects are associated with such a treatment [16]. Therefore, safe and effective therapy for OA is urgently needed to be developed. It has been reported that Foxo3a expression is altered in various types of inflammatory diseases [17]. To our knowledge, our current study is the first to demonstrate that Foxo3a is highly expressed in IL- $1 \beta$-treated chondrocytes compared with control chondrocytes, suggesting the Foxo3a might be a potential biomarker for OA.

Inducible NOS expression is induced by various factors such as inflammatory cytokines (IL-1 $\beta$, IL-17, TNF- $\alpha$ and IFN- $\gamma$ ) [18], bacterial infection [19] and degradation of extracellular matrix [20]. The upstream targets of iNOS expression are associated with three main regulators including NF- $\kappa \mathrm{B}$, STAT-1 and MAPKs signaling pathways [21]. More specifically, IL- $1 \beta$-induced OA is mainly associated with translocation of NF- $\kappa \mathrm{B}$ into nucleus, where it binds to the promotor region of iNOS [22]. Subsequently, iNOS efficiently catalyzes the production of nitric oxide (NO) which plays a critical role in the pathogenesis of OA [23]. It has been reported that iNOS-mediated NO production in OA chondrocytes is the leading cause of cartilage destruction, and depletion of iNOS in mice inhibited the development of OA [24]. Consistently, we found in our study that iNOS expression was significantly increased in IL- $1 \beta$ -treated chondrocytes as compared to the control cells. It was demonstrated that NO synthesis was actively progressed in OA chondrocytes [25]. In other words, OA chondrocytes might undergo some cellular events like inflammatory response, apoptosis or oxidative stress [26]. Moreover, increased expression of iNOS and NO have been found in vivo in inflammatory, septic arthritis and in patients with rheumatoid arthritis [27]. Meanwhile, our data showed that enhancement of iNOS expression was suppressed by down-regulation of Foxo3a what suggests that Foxo3a could be a new clinical target for OA treatment and also for other iNOS-related inflammatory diseases.
Chondrocytes apoptosis is also regarded as the key factor in OA progression. Nitric oxide generation is known as the primary executioner of chondrocytes apoptosis through regulating caspase-3-dependent signaling [28]. Mechanically, NO-induced chondrocytes apoptosis is associated with mitochondrial dysfunction, DNA damage and iNOS signaling [29]. In this study, we provided evidence that down-regulated Foxo3a inhibited chondrocytes apoptosis through regulating caspase-3-dependent signaling, indicating that inhibition of Foxo3a expression offers a clinically-relevant perspective for the treatment of OA by regulating chondrocytes' apoptosis. However, the detailed mechanism of this phenomenon should be clarified in future study. Recently, many researchers point out that oxidative stress is a critical risk factor for the onset of OA [30]. ROS, as the mediators of oxidative stress, not only participate in the process of cell matrix degradation but also control chondrocytes' life cycle during OA [30]. On the other hand, Foxo transcription factors have been implicated in various ROS-related processes including cellular aging, proliferation, senescence or apoptosis [31]. For instance, mammalian Sterile 20-like kinase 1 (MST-1) mediates ROS-induced neuronal apoptosis via Foxo3a phosphorylation [32]. Foxo3a also suppressed ROS-induced apoptosis in differentiated 3T3-L1 adipocytes [33]. Hence, the mechanism underlying the protective effect of down-regulated Foxo3a on chondrocytes' apoptosis might be also associated with ROS-mediated signaling.

Tenascin-c is ECM glycoprotein which increased levels were found in the synovial fluid in OA patients [34]. Production of Tnc can also induce inflammatory mediators and promote matrix degradation in OA, and its levels could serve as a biomarker of joint damage and a trigger of further joint degradation [35, 36]. In our in vitro system, the level of Tnc was significantly increased in OA chondrocytes compared with control chondrocytes, which was related to Foxo3a expression. Therefore, these results suggest that Foxo3a is critically involved in the production of Tnc during OA. In other words, Foxo3a may regulate the OA-mediated inflammation via modulating Tnc. Furthermore, extracellular addition of Tnc abrogated the effects of Foxo3a knockdown on apoptosis and iNOS expression in IL- $1 \beta$-treated chondrocytes, suggesting that Tnc promoted apoptosis in IL- $1 \beta$-mediated OA chondrocytes. On the contrary, the protective effect of Tnc on human OA cartilage was observed in an in vitro system [37]. It was shown in $\mathrm{Tnc}^{-/}$mice that cartilage repair was significantly slower than that in wild type mice, and cartilage degeneration was enhanced by deficiency of Tnc [38]. Furthermore, 
Matsui's group reported that deficiency of Tnc delayed articular cartilage repair in vivo and treatment of full-thickness osteochondral defects by filling the defect with exogenous Tnc results in the inhibition of cartilage degeneration [39]. These different results may be affected by experimental model, experimental object or experimental conditions. In spite of this, the exact mechanisms of how Tnc plays dual roles in OA remain unclear, and needs further research.

In the present study, we provided evidence that down-regulated Foxo3a protects in vitro against IL-1 $\beta$ -mediated inflammation and IL- $1 \beta$-induced chondrocytes' apoptosis. These results also revealed that those protective effects of down-regulated Foxo3a on OA were blocked by extracellular addition of Tnc, indicating that down-regulation of Foxo3a exhibited the potent cytoprotective activity in the in vitro $\mathrm{OA}$ model via modulating Tnc. Our study suggests potential usefulness of targeting Foxo3a as a candidate for the development of a novel and effective treatment for OA.

\section{Funding}

This work was supported by Zhejiang Provincial Basic Public Welfare Research Project (Grant No. LGF19H7000), Zhejiang Provincial Medical and Health Research Surface Project (Grant No. 2018KY777) and Zhejiang Traditional Chinese Medicine Technology Project (Grant No. 2017ZA134).

\section{Competing interests}

The authors declare that they have no competing interests.

\section{Availability of data and materials}

All data generated or analyzed during this study are included in this published article.

\section{Authors' contribution}

FW and QBW conceived and designed the experiments, MZ analyzed and interpreted the results of the experiments, QS performed the experiments.

\section{Ethics approval and consent to participate}

The animal use protocol listed below has been reviewed and approved by the Animal Ethical and Welfare Committee. Approval No. 2019008.

\section{References}

1. Nelson AE. Osteoarthritis year in review 2017: clinical. Osteoarthritis Cartilage. 2018; 26(3): 319-325, doi: 10.1016/j. joca.2017.11.014, indexed in Pubmed: 29229563.
2. Blagojevic M, Jinks C, Jeffery A, et al. Risk factors for onset of osteoarthritis of the knee in older adults: a systematic review and meta-analysis. Osteoarthritis Cartilage. 2010; 18(1): 24-33, doi: 10.1016/j.joca.2009.08.010, indexed in Pubmed: 19751691.

3. Squires GR, Okouneff S, Ionescu M, et al. The pathobiology of focal lesion development in aging human articular cartilage and molecular matrix changes characteristic of osteoarthritis. Arthritis Rheum. 2003; 48(5): 1261-1270, doi: 10.1002/ art.10976, indexed in Pubmed: 12746899.

4. Goldring MB, Otero M. Inflammation in osteoarthritis. Curr Opin Rheumatol. 2011; 23(5): 471-478, doi: 10.1097/ BOR.0b013e328349c2b1, indexed in Pubmed: 21788902.

5. Blanco FJ, Rego I, Ruiz-Romero C. The role of mitochondria in osteoarthritis. Nat Rev Rheumatol. 2011; 7(3): 161-169, doi: 10.1038/nrrheum.2010.213, indexed in Pubmed: 21200395.

6. Bjarnason I, Scarpignato C, Holmgren E, et al. Mechanisms of Damage to the Gastrointestinal Tract From Nonsteroidal Anti-Inflammatory Drugs. Gastroenterology. 2018; 154(3): 500-514, doi: 10.1053/j.gastro.2017.10.049, indexed in $\mathrm{Pu}-$ bmed: 29221664.

7. Chiquet-Ehrismann R. Tenascins, a growing family of extracellular matrix proteins. Experientia. 1995; 51(9-10): 853-862, doi: 10.1007/bf01921736, indexed in Pubmed: 7556567.

8. Whitby DJ, Longaker MT, Harrison MR, et al. Rapid epithelialisation of fetal wounds is associated with the early deposition of tenascin. J Cell Sci. 1991; 99 ( Pt 3): 583-586, indexed in Pubmed: 1719005.

9. Tsunoda T, Inada H, Kalembeyi I, et al. Involvement of large tenascin-C splice variants in breast cancer progression. Am J Pathol. 2003; 162(6): 1857-1867, doi: 10.1016/S00029440(10)64320-9, indexed in Pubmed: 12759243.

10. Sofat N, Robertson SD, Hermansson M, et al. Tenascin-C fragments are endogenous inducers of cartilage matrix degradation. Rheumatol Int. 2012; 32(9): 2809-2817, doi: 10.1007/ s00296-011-2067-8, indexed in Pubmed: 21874326.

11. Yoshimura E, Majima A, Sakakura Y, et al. Expression of tenascin- $\mathrm{C}$ and the integrin alpha 9 subunit in regeneration of rat nasal mucosa after chemical injury: involvement in migration and proliferation of epithelial cells. Histochem Cell Biol. 1999; 111(4): 259-264, doi: 10.1007/s004180050356, indexed in Pubmed: 10219625.

12. Midwood K, Sacre S, Piccinini AM, et al. Tenascin-C is an endogenous activator of Toll-like receptor 4 that is essential for maintaining inflammation in arthritic joint disease. Nat Med. 2009; 15(7): 774-780, doi: 10.1038/nm.1987, indexed in Pubmed: 19561617.

13. Nho RS, Hergert P. FoxO3a and disease progression. World J Biol Chem. 2014; 5(3): 346-354, doi: 10.4331/wjbc.v5.i3.346, indexed in Pubmed: 25225602.

14. Turrel-Davin F, Tournadre A, Pachot A, et al. FoxO3a involved in neutrophil and $\mathrm{T}$ cell survival is overexpressed in rheumatoid blood and synovial tissue. Ann Rheum Dis. 2010; 69(4): 755-760, doi: 10.1136/ard.2009.109991, indexed in Pubmed: 19435720.

15. Johnson CI, Argyle DJ, Clements DN. In vitro models for the study of osteoarthritis. Vet J. 2016; 209: 40-49, doi: 10.1016/j. tvj1.2015.07.011, indexed in Pubmed: 26831151.

16. Nakata K, Hanai T, Take Y, et al. Disease-modifying effects of COX-2 selective inhibitors and non-selective NSAIDs in osteoarthritis: a systematic review. Osteoarthritis Cartilage. 2018; 26(10): 1263-1273, doi: 10.1016/j.joca.2018.05.021, indexed in Pubmed: 29890262.

17. Peng SL. Forkhead transcription factors in chronic inflammation. Int J Biochem Cell Biol. 2010; 42(4): 482-485, doi: 10.1016/j.biocel.2009.10.013, indexed in Pubmed: 19850149. 
18. Soufli I, Toumi R, Rafa H, et al. Overview of cytokines and nitric oxide involvement in immuno-pathogenesis of inflammatory bowel diseases. World J Gastrointest Pharmacol Ther. 2016; 7(3): 353-360, doi: 10.4292/wjgpt.v7.i3.353, indexed in Pubmed: 27602236.

19. Rodrigues JP, Caldas IS, Gonçalves RV, et al. S. mansoni-T. cruzi co-infection modulates arginase-1/iNOS expression, liver and heart disease in mice. Nitric Oxide. 2017; 66: 43-52, doi: 10.1016/j.niox.2017.02.013, indexed in Pubmed: 28268114.

20. Adler N, Schoeniger A, Fuhrmann H. Effects of transforming growth factor- and interleukin-1 on inflammatory markers of osteoarthritis in cultured canine chondrocytes. Am J Vet Res. 2017; 78(11): 1264-1272, doi: 10.2460/ajvr.78.11.1264, indexed in Pubmed: 29076366.

21. Berenbaum F. Signaling transduction: target in osteoarthritis. Curr Opin Rheumatol. 2004; 16(5): 616-622, doi: 10.1097/01. bor.0000133663.37352.4a, indexed in Pubmed: 15314504.

22. Wang SN, Xie GP, Qin CH, et al. Aucubin prevents interleukin-1 beta induced inflammation and cartilage matrix degradation via inhibition of NF- B signaling pathway in rat articular chondrocytes. Int Immunopharmacol. 2015; 24(2): 408-415, doi: 10.1016/j.intimp.2014.12.029, indexed in $\mathrm{Pu}-$ bmed: 25576403.

23. Studer R, Jaffurs D, Stefanovic-Racic M, et al. Nitric oxide in osteoarthritis. Osteoarthritis Cartilage. 1999; 7(4): 377-379, doi: 10.1053/joca.1998.0216, indexed in Pubmed: 10419772.

24. Xie Q, Nathan C. The high-output nitric oxide pathway: role and regulation. J Leukoc Biol. 1994; 56(5): 576-582, doi: 10.1002/jlb.56.5.576, indexed in Pubmed: 7525816.

25. Jung YK, Park HR, Cho HJ, et al. Degrading products of chondroitin sulfate can induce hypertrophy-like changes and MMP-13/ADAMTS5 production in chondrocytes. Sci Rep. 2019; 9(1): 15846, doi: 10.1038/s41598-019-52358-4, indexed in Pubmed: 31676809.

26. Yui N, Yudoh K, Fujiya H, et al. Mechanical and oxidative stress in osteoarthritis. The Journal of Physical Fitness and Sports Medicine. 2016; 5(1): 81-86, doi: 10.7600/jpfsm.5.81.

27. Dey P, Panga V, Raghunathan S. A cytokine signalling network for the regulation of inducible nitric oxide synthase expression in rheumatoid arthritis. PLoS One. 2016; 11(9): e0161306, doi: 10.1371/journal.pone.0161306, indexed in Pubmed: 27626941.

28. Hwang HS, Kim HAh. Chondrocyte apoptosis in the pathogenesis of osteoarthritis. Int J Mol Sci. 2015; 16(11): 26035 -26054, doi: 10.3390/ijms161125943, indexed in Pubmed: 26528972.
29. Moncada S, Erusalimsky JD. Does nitric oxide modulate mitochondrial energy generation and apoptosis? Nat Rev Mol Cell Biol. 2002; 3(3): 214-220, doi: 10.1038/nrm762, indexed in Pubmed: 11994742.

30. Lepetsos P, Papavassiliou KA, Papavassiliou AG. Redox and NF- B signaling in osteoarthritis. Free Radic Biol Med. 2019; 132: 90-100, doi: 10.1016/j.freeradbiomed.2018.09.025, indexed in Pubmed: 30236789.

31. Storz P. Forkhead homeobox type O transcription factors in the responses to oxidative stress. Antioxid Redox Signal. 2011; 14(4): 593-605, doi: 10.1089/ars.2010.3405, indexed in Pubmed: 20618067.

32. Yuan Z, Lehtinen MK, Merlo P, et al. Regulation of neuronal cell death by MST1-FOXO1 signaling. J Biol Chem. 2009; 284(17): 11285-11292, doi: 10.1074/jbc.M900461200, indexed in Pubmed: 19221179.

33. Kojima T, Norose T, Tsuchiya K, et al. Mouse 3T3-L1 cells acquire resistance against oxidative stress as the adipocytes differentiate via the transcription factor FoxO. Apoptosis. 2010; 15(1): 83-93, doi: 10.1007/s10495-009-0415-x, indexed in Pubmed: 19842039.

34. Hasegawa M, Nakoshi Y, Muraki M, et al. Expression of large tenascin-C splice variants in synovial fluid of patients with rheumatoid arthritis. J Orthop Res. 2007; 25(5): 563-568, doi: 10.1002/jor.20366, indexed in Pubmed: 17262825.

35. Aungier SR, Cartwright AJ, Schwenzer A, et al. Targeting early changes in the synovial microenvironment: a new class of immunomodulatory therapy? Ann Rheum Dis. 2019; 78(2): 186-191, doi: 10.1136/annrheumdis-2018-214294, indexed in Pubmed: 30552174.

36. Chockalingam PS, Glasson SS, Lohmander LS. Tenascin-C levels in synovial fluid are elevated after injury to the human and canine joint and correlate with markers of inflammation and matrix degradation. Osteoarthritis Cartilage. 2013; 21(2): 339-345, doi: 10.1016/j.joca.2012.10.016, indexed in Pubmed: 23142724.

37. Unno H. Prevention mechanism of tenascin-C for the cartilage degeneration. Jap Journal of Joint Diseases. 2016; 35(2): 131-136, doi: 10.11551/jsjd.35.131.

38. Okamura N, Hasegawa M, Nakoshi Y, et al. Deficiency of tenascin-C delays articular cartilage repair in mice. Osteoarthritis Cartilage. 2010; 18(6): 839-848, doi: 10.1016/j. joca.2009.08.013, indexed in Pubmed: 19747998.

39. Matsui $Y$, Hasegawa M, Iino T, et al. Tenascin-C prevents articular cartilage degeneration in murine osteoarthritis models. Cartilage. 2018; 9(1): 80-88, doi: 10.1177/1947603516681134, indexed in Pubmed: 29219023.

Submitted: 16 July, 2019

Accepted after reviews: 30 December, 2019 Available as AoP: 31 January, 2020 